\title{
The effect of psychological distance on young children's future predictions
}

\author{
Tessa R. Mazachowsky (i) । Christine Koktavy I Caitlin E.V. Mahy
}

Department of Psychology, Brock University, St Catharines, Canada

\section{Correspondence}

Tessa Mazachowsky, Department of Psychology, Brock University, 1812 Sir Isaac Brock Way, St Catharines, ON L2S 3A1

Canada.

Email: tm10nt@brocku.ca

Funding information NSERC, Grant/Award Number: RGPIN-201503774

\begin{abstract}
The current study examined the impact of psychological distance on children's performance on the pretzel task. In this task, children eat pretzels (inducing thirst) and then are asked to reason about future preferences (pretzels or water). Children typically perform poorly on this task, indicating a future preference for water over pretzels, potentially due to conflicting current and future states. Given past work showing that children's future reasoning is more accurate for another person, we asked 90 thirsty 3- to 7year-olds to reason about their own and an experimenter's future preference. Results showed that thirsty children had more difficulty predicting their own future preference compared with the experimenter's. Thirstier children were more likely to predict a future preference for water. Thirst interacted with age when making a future choice for the experimenter. How psychological distance might boost episodic foresight and possible reasons for children's poor pretzel task performance are discussed.
\end{abstract}

\section{Highlights}

- Does psychological distancing improve children's ability to make accurate future predictions when current and future states conflict?

- Using the Pretzel task, thirsty children were less accurate when predicting their own future preferences compared with the future preferences of another person. 
- Psychological distancing may help children overcome their current state to reason more accurately about the future.

\section{KEYWORDS}

episodic foresight, future reasoning, physiological state, psychological distance, self-other differences, young children

\section{1 | INTRODUCTION}

Episodic foresight is the ability to project oneself into future scenarios in order to pre-experience future events. In recent years, this ability has received increased interest particularly in its emergence and early development (e.g., Atance \& Jackson, 2009; Hudson, Mayhew, \& Prabhakar, 2011; Russell, Alexis, \& Clayton, 2010). A substantial body of research now exists showing that episodic foresight undergoes significant development between 3 and 7 years old, with much progress by 5 years of age (e.g., Atance \& Meltzoff, 2005; Suddendorf, Nielsen, \& von Gehlen, 2011). For example, in the Picture-Book task, children are asked to imagine visiting a future location (e.g., desert) and then asked to choose one of three items that they would need for that location (e.g., sunglasses, soap, or a seashell; Atance \& Meltzoff, 2005). In this task, age-related increases are found between 3 and 7 years old, with older children selecting the appropriate item to bring with them more often than younger children. One notable exception to this developmental pattern is for an episodic foresight task that requires children to predict a future state that is in conflict with a current physiological state. Under these circumstances, there are often no age-related increases, with children 3 to 7 years old performing equally poorly (Atance \& Meltzoff, 2006; Mahy, 2016; Mahy, Grass, Wagner, \& Kliegel, 2014).

Anticipating the future may be difficult for humans and non-human animals alike when current and future states conflict. The Bischof-Kohler hypothesis posits that non-human animals cannot anticipate needs that are not currently experienced (Bischof-Kohler, 1985). This powerful influence of current states on future behaviour similarly exists in adult humans and is known as the Presentism bias (Gilbert, Gill, \& Wilson, 2002). Gilbert et al. (2002) suggest that an individual's current state can interfere with their ability to accurately project themselves forward in time to predict or imagine future experiences. Further, visceral states such as hunger, thirst, or emotion may be particularly difficult to overcome and may lead to attentional narrowing to a current state at the cost of accurate future reasoning (Loewenstein, 1996). For example, hungry adult grocery shoppers were found to overestimate their future food needs and buy more food than sated grocery shoppers (Gilbert et al., 2002; Nisbett \& Kanouse, 1969).

Research examining the effect of current-future state conflict in children consistently shows that children struggle to reason accurately about their future preferences. The pretzel task (Atance \& Meltzoff, 2006) is one episodic foresight task that has been used to investigate children's ability to reason about the future when experiencing a conflicting current state. In this task, children are first asked about their baseline preference between pretzels and water, with the majority of children reporting a preference for pretzels. However, after children consume pretzels and thus are thirsty, children who previously reported a preference for pretzels at baseline tend to report a future preference for water (Atance \& Meltzoff, 2006; Mahy, 2016). Further, Atance and Meltzoff (2006) found that children in a control condition, who did not consume pretzels (and thus were not thirsty), reported that they would prefer pretzels for the future significantly more than children who consumed pretzels. At the end of this task, children are generally offered water to drink and the amount that they drink is measured to operationalize thirst. Past work has shown that the amount of water children drink is positively related to their future preference for water (Atance \& Meltzoff, 2005; 
Mahy, 2016) and that non-thirsty children (who do not eat pretzels) drink less water than children who have consumed pretzels (Atance \& Meltzoff, 2006). Notably, no developmental increases have been documented in the Pretzel task from age three to adulthood (Atance \& Meltzoff, 2006; Cheke \& Clayton, 2019; Kramer, Goldfarb, Tashjian, \& Lagattuta, 2017; Mahy, 2016). This lack of age-related change in Pretzel task performance suggests that both children and adults' episodic foresight is negatively affected by direct conflict between a current and future state. Though both children and adults struggle to forecast the future when experiencing a conflicting current state, research has yet to investigate if children's ability to reason for the future in the face of current-future state conflict can be improved. Thus, it is unknown whether children can be helped to overcome the powerful influence of a current state to reason more accurately about the future.

One promising approach to increase accurate reasoning about the future is to manipulate psychological distance to observe whether children reason differently about a thirsty self's future compared with a thirsty other's future. Research examining the ability to mentally detach from one's own beliefs, desires, and circumstances to reason from a perspective different from one's own, known as psychological distancing, reports positive effects on children's and adults' future decision making in a number of future-oriented domains (e.g., Kross \& Grossmann, 2012; Lee \& Atance, 2016; Prencipe \& Zelazo, 2005; Pronin, Olivola, \& Kennedy, 2008; White \& Carlson, 2016; Ziegler \& Tunney, 2012).

Studies with adults have found that taking another person's perspective (compared with one's own) results in improved reasoning abilities and decision making (e.g., Grossmann \& Kross, 2014; Pronin et al., 2008). For instance, Grossmann and Kross (2014) found that participants reasoned more wisely when answering questions about infidelity in a friend's romantic relationship compared with their own relationship. Similarly, Bauckham et al. (2018) found that when participants considered future preferences for themselves, a close other, and a generic peer, young adults displayed a presentism bias for themselves (i.e., predicted less change between current and future preferences) but a smaller presentism bias as psychological distance increased (i.e., predicted more change between current and future preferences for generic peers compared with a close other).

Similarly, research on psychological distancing in children generally shows that 3- to 5-year-olds are able to reason about the future more accurately when adopting a third-person perspective or the perspective of a peer compared with reasoning about their own future (Bélanger, Atance, Varghese, Nguyen, \& Vendetti, 2014; Lee \& Atance, 2016; Russell et al., 2010). For example, Bélanger et al. (2014) examined children's understanding of changes in preferences over time (e.g., preferring Kool-Aid as a child and coffee as an adult) and found that 3- to 5-year-olds were better able to predict a peer's adult preference compared with their own adult preference. In addition, Lee and Atance (2016) found that 3-and 4-year-old children had difficulty reasoning about their own future preferences but were better at reasoning about future preferences of similar or dissimilar peers. Taken together, these findings suggest that taking a more psychologically distant perspective can improve decision making and reasoning in both children and adults. Accordingly, psychological distancing may allow children to take a more neutral perspective and detach from any current mental, emotional, or physiological state that would otherwise influence their thinking (Lee \& Atance, 2016). Thus, it follows that psychological distancing might improve children's ability to disengage from their own current state of thirst when considering future preferences of another person compared with themselves.

The current study was designed to examine the effect of psychological distance on children's ability to make future predictions when experiencing a conflicting current state. Children between 3 and 7 years old completed the pretzel task for themselves and for the experimenter (counterbalanced). First, we expected children to be more accurate in predicting the experimenter's future preference compared with their own, given past work documenting the benefits of taking a psychologically distant perspective (e.g., Bélanger et al., 2014; Lee \& Atance, 2016). Second, in line with past work, we predicted that there would be no age-related increases in performance on the pretzel task for self or other (e.g., Atance \& Meltzoff, 2006; Cheke \& Clayton, 2019; Mahy et al., 2014). Third, we expected that children's thirst would be related to their own future preference but not the experimenter's future preference. That is, children's current level of thirst should exert a larger influence on reasoning about their own future preferences (Mahy, 2016) compared with another person's future preferences due to the salience of one's own current physiological state. 


\section{2 | METHOD}

\section{1 | Participants}

Participants were 100 typically developing children aged three to seven (24 3-year-olds, 21 4-year-olds, 18 5-yearolds, 19 6-year-olds, and 18 7-year-olds). Ten children were excluded from the analyses for uncooperativeness $(n=4)$ or failure to eat a sufficient number of pretzels to induce thirst $(n=6$; see Mahy et al., 2014). The final sample consisted of 90 children: 18 3-year-olds ( 9 girls; $M=42.89, S D=3.18$ ), 18 4-year-olds, ( 9 girls; $M=52.78, S D=3.77$ ), 18 5-year-olds ( 9 girls; $M=67.00, S D=3.01$ ), 186 -year-olds ( 9 girls; $M=78.56, S D=3.20$ ), and 187 -year-olds $(9$ girls; $M=88.72, S D=3.69$ ). Given the repeated measures design of the study, this sample size was sufficient to detect a medium effect size and was in line with past studies using the same task (e.g., Mahy, 2016). Children were predominantly Caucasian (83.7\%) and from a middle class background, with $67 \%$ of parents reporting holding at least a university degree and $52 \%$ of parents reporting a household income above 75,000 dollars. Children were recruited from university participant databases at the University of Ottawa and the University of Oregon.

\section{2 | Pretzel task}

The Pretzel Task (adapted from Atance \& Meltzoff, 2006). Children were offered a small amount of apple juice (3 oz.) at the beginning of this task to ensure they were not thirsty at baseline. After children had sufficient opportunity to drink some juice, it was removed and the experimenter asked what the child preferred to have right now: pretzels to eat or water to drink. Children were simultaneously shown a colour photograph of a bowl of pretzels and a bottle of water. Children who indicated a preference for water were excluded from the analysis. Regardless of children's initial preference, the experimenter then told the child that she liked pretzels and, if given a choice, she would choose pretzels right now (to establish the experimenter's baseline preference). Then, the experimenter told children that it was time to listen to a story and that the child could eat as many pretzels as they liked during this time. A small bowl with 36 pretzel sticks was placed in front of the child and the experimenter read a story ("The Perfect Picnic"; Maestro, 1986), which took approximately 6 min.

After the story was finished, the experimenter removed the bowl of pretzel sticks. Then, the experimenter asked children to pretend that they were going to come back to the laboratory the next day to play a marble game (children were shown a photograph of the marble game). It was emphasized that children were not going to play with the marble game today but needed to imagine playing with it tomorrow. Children were shown the photograph of a bowl of pretzels and a bottle of water and were asked what they would like to have during the marble game tomorrow (selffuture question): pretzels to eat or water to drink? Children were also asked to pretend that the experimenter was going to come back tomorrow to play with the marble game and, like them, the experimenter had just eaten some pretzels. Children were asked what the experimenter would like to have tomorrow (other-future question): pretzels to eat or water to drink? The order in which children were asked the self-future question and the other-future question was counterbalanced.

After children had answered questions about their own and the experimenter's future preferences, they were offered 9 oz. of water to drink. The amount of water (0-9 oz.) children drank was recorded (as a measure of thirst), as was the number of pretzels children ate. Only children who ate at least 8 pretzels (following a cut-off established as a minimum to induce thirst by Mahy, 2016) and indicated an initial preference for pretzels over water were included in the analysis $(N=69)$ to ensure that a preference for water after eating pretzels did not simply reflect a baseline preference for water over pretzels. Children were given a score of 1 if they indicated that they or the experimenter would prefer pretzels tomorrow and a score of 0 if they said that they or the experimenter would prefer water tomorrow. 


\section{3 | Procedure}

Children were tested in a 60-min laboratory session that measured several episodic foresight tasks that were not related to the current research question and thus not reported here. The pretzel task was always the first task administered. Children were randomly assigned to one of two counterbalancing orders of the pretzel task: one in which they received the self-future question followed by the other-future question and the other in which they received the other-future question before the self-future question. At the end of the session, children were thanked and were given a small prize for their participation. The research ethics boards at University Ottawa and University Oregon approved all procedures.

\section{3 | RESULTS}

Initially, 69 children ( $M=64.39$ months, $S D=17.13$, range = 36 to 96 months) indicated a baseline preference for pretzels, whereas 21 children indicated a baseline preference for water. There were no significant differences between these groups of children on parent ethnicity, education, and income level, ps >0.05. Only children who indicated a baseline preference for pretzels were included in the analyses $(N=69)$ given that subsequent questions in this task were based on the assumption that children initially preferred pretzels. Further, given that children were told that the experimenter's baseline preference was for pretzels over water, we wanted to ensure that only children who expressed a preference for pretzels at baseline were included to equalize the two conditions as much as possible. Children ate a large number of pretzels on average $(M=18.56, S D=7.90)$ indicating that our thirst manipulation was successful. At the end of the task, children drank approximately 2 oz. of water, on average, when it was offered $(M=2.06, S D=2.10)$. Children who ate more pretzels also drank more water at the end of the task, $r(67)=0.32$, $p=0.007$.

Table 1 shows children's mean performance on the pretzel task by age group and perspective (self vs. other). The dependent variables were the child's future choice for self and other (water scored as 0 and pretzels scored as 1). A McNemar's test showed that children chose pretzels over water significantly more often for the experimenter (52\%) than for themselves (32\%; $p=0.02$ ). Thus, children performed better when making future predictions for another person compared with themselves. To examine whether children's performance differed from chance, a binomial test was conducted on pretzel task performance for self and other. Children were significantly worse than chance when making a future choice for themselves (32\%; $p=0.004)$, whereas children's future choice for the experimenter did not significantly differ from chance $(52 \% ; p=0.81)$.

Preliminary analyses of the effect of gender $(B=0.15, p=0.78)$ and counterbalancing order $(B=0.57, p=0.28)$ were non-significant predictors of choice for self, so these factors were excluded from further analyses. For children's future choice for themselves, we used a logistic regression where child's age in months, thirst, and an age by thirst interaction were entered as predictors of children's pretzel task performance. Thirst significantly predicted children's performance for self, Wald $\chi^{2}(1)=4.08, p=0.04$. Thirstier children tended to predict that their future preference would be water over pretzels. Children's age in months (Wald $\chi^{2}[1]=0.86, p=0.36$ ) and the age by thirst interaction (Wald $\chi^{2}[1]=0.04, p=0.84$ ) were not significant predictors of children's performance for self.

TABLE 1 Means and standard deviations of pretzel task performance by age group and perspective

\begin{tabular}{llllll} 
& \multicolumn{1}{l}{ Age group } \\
\cline { 2 - 6 } Perspective & 3 & 4 & 5 & 6 & 7 \\
Self & $0.50(0.52)$ & $0.27(0.46)$ & $0.31(0.48)$ & $0.42(0.51)$ & $0.08(0.28)$ \\
Other & $0.47(0.52)$ & $0.67(0.49)$ & $0.58(0.51)$ & $0.42(0.51)$ & $0.46(0.52)$ \\
\hline
\end{tabular}


Preliminary analyses of the effect of gender $(B=0.14, p=0.79)$ and counterbalancing order $(B=-0.43, p=0.39)$ were non-significant predictors of choice for the experimenter, so these factors were excluded from further analyses. For children's future choice for other, we again used a logistic regression where age in months, thirst, and an age by thirst interaction were entered as predictors of children's performance on the pretzel task. Children's age (Wald $\chi^{2}$ $[1]=1.52, p=0.22$ ) and thirst (Wald $\chi^{2}[1]=0.32, p=0.57$ ) were not significant predictors of children's performance on the future other question. The interaction between children's age and their thirst level was a significant predictor of performance, Wald $\chi^{2}[1]=6.21, p=0.01$. To examine this interaction, we compared children who were in the bottom quartile (least thirsty) and top quartile (most thirsty). Results showed that children who were low in thirst performed similarly when making a choice for another person in the future regardless of age $\left(\right.$ Wald $\chi^{2}[1]=2.17$, $p=0.14$ ), whereas children who were high in thirst performed worse when making a choice for another person as they got older (choosing water as the future preference for other more than pretzels with age; Wald $\chi^{2}[1]=4.51$, $p=0.03)$.

\section{4 | DISCUSSION}

Current results showed that the perspective from which children made future choices mattered. Children had difficulty overcoming their present state of thirst to make accurate predictions about their own future preferences but experienced less difficulty when making future predictions for the experimenter. Interestingly, children's prediction for the experimenter's future desires did not differ from chance, but they were significantly below chance when predicting their own future desires. Further, children's age in months did not predict performance on the pretzel task for self. Finally, children's level of thirst predicted their own future preference for water and interacted with age when predicting another person's future preference.

The current study found an other-over-self advantage in the pretzel task, suggesting that taking a more psychologically distanced perspective may help children detach from the influence of a current conflicting state. By considering future preferences from a more distant perspective, children may have been able to momentarily escape from their own state of thirst to better predict the experimenter's previously stated preference for pretzels over water. Thus, children's current state did not have as powerful of an influence on their predictions for another person compared with their predictions for themselves. Other studies have similarly documented children's superior episodic foresight when psychological distance is increased (e.g., Bélanger et al., 2014; Lee \& Atance, 2016; Russell et al., 2010). In general, psychological distancing may help children improve their accuracy for future predictions, even when experiencing a conflicting current state. However, predicting another's future preference did not result in anywhere near perfect accuracy and in fact children performed at chance levels when doing so.

Importantly, differences in children's future predictions for self and other are relevant to two explanations for children's difficulty on the pretzel task. On the one hand, children's struggle with future reasoning might be due to their current state (e.g., thirst) being in direct conflict with a future state (i.e., the current-future state conflict explanation; Atance \& Meltzoff, 2005; Mahy, 2016; Mahy et al., 2014). Research in support of the current-future state conflict explanation shows that although children can accurately reason about the future in the absence of a conflicting current state (e.g., developmental increases in performance on the Picture-Book task), they have much more difficulty when there is a conflicting current state (e.g., no developmental change in pretzel task performance; Mahy et al., 2014). Our current findings are somewhat in line with this explanation. We found that thirsty children struggled more in predicting their own future preference compared with the experimenter's future preference when a current state (i.e., thirst) was in conflict with a future state (i.e., a general preference for pretzels). However, by this account, it is possible that a current state of thirst could also interfere with children's ability to make accurate predictions for another person.

On the other hand, the physiological misunderstanding explanation predicts that children's poor performance on the pretzel task is driven by a general lack of understanding of how physiological states change over time (rather than a conflict between current and future states). In support of this explanation, Moses, Coon, and Wusinich (2000) 
showed that 3- to 5-year-olds had a poor understanding of how physiological desires change over time (e.g., desire for water after drinking a long time ago versus recently). Interestingly, children did not display this same difficulty understanding changes in perception (e.g., knowledge of a box's contents after opening versus not opening the box) or attitude-based desires (e.g., desire to play with a dog after a negative experience versus a positive experience) over time. According to this explanation, children should struggle with predicting future preferences of self and other equally if their performance is driven by a general lack an understanding of physiological states. In the current study, low levels of performance on the pretzel task for self and other could, at least partially, be explained by children's general lack of understanding of physiological states. Yet children's at chance performance on the pretzel task for other compared with below chance performance for self suggest some support for the current-state bias explanation (e.g., Kramer et al., 2017; Mahy, 2016).

Although the current study is unable to directly test these two explanations, findings suggest that physiological states may be difficult for children to understand overall and may be increasingly difficult to predict when a current physiological state conflicts with a future state. Direct testing of these two explanations could be explored in future work by including a control condition where children reason about future physiological states in the absence of thirst (i.e., when current-future state conflict is not present) to see if they still have difficulty making accurate future decisions for self and other. If children still struggle with predicting future states in the absence of a conflicting state, this would suggest stronger support for the physiological misunderstanding explanation.

As expected, no effect of age on pretzel task performance was found when 3- to 7-year-olds were asked to predict their own future preference. This finding is consistent with past studies that have also found no age-related improvements in pretzel task performance between 3 years old and young adulthood (Atance \& Meltzoff, 2006; Cheke \& Clayton, 2019; Kramer et al., 2017; Mahy et al., 2014). Interestingly, many other episodic foresight tasks where present and future states do not conflict show age-related improvements during the preschool years (e.g., Atance \& Jackson, 2009; Suddendorf \& Busby, 2005), suggesting that current-future state conflict may pose special difficulty for making accurate future predictions.

When children were less thirsty (i.e., current and future state conflict was reduced), they were better able to reason for themselves in the future, compared with when they were thirstier (i.e., when current and future state conflicted). The impact of thirst on children's future reasoning demonstrates that a current state of thirst influenced their ability to accurately reason for themselves in the future. Overall, children's difficulty with current-future state conflict is consistent with previous studies that show thirsty children anticipate preferring water in the future, despite reporting a preference for pretzels prior to experiencing thirst (e.g., Atance \& Meltzoff, 2006; Mahy, 2016). Mahy (2016) also found that when children's thirst was quenched, by providing them with water to drink (and thus alleviated the conflicting state), they reverted to their baseline preference for pretzels in the future. Thus, the current findings support that children are poor at thinking about future preferences particularly when they are in conflict with a current physiological state.

Interestingly, when children were asked to make a future preference choice for the experimenter, age and thirst interacted to predict children's performance. Children who were the least thirsty performed similarly across age, whereas children who were the thirstiest tended to perform worse with age. Seemingly, thirsty older children had more difficulty overcoming their current state and let their current state of thirst influence their choice for another person in the future more than thirsty younger children. This effect was unexpected given that the current state was not predicted to influence children's ability to make decisions for another person. Future research is needed to determine if this effect can be replicated.

There are several limitations of the current study that should be acknowledged. First, the experimenter did not eat pretzels alongside the child and was therefore not experiencing a current state of thirst like that of the child. Although the experimenter expressed a baseline preference for pretzels and children were instructed to imagine that the experimenter had eaten pretzels, actual consumption of pretzels by the experimenter would have likely made it easier for the child to understand that the experimenter was thirsty. Additionally, the added demand of needing to imagine the experimenter's current state of thirst could have led children to base their prediction for the 
experimenter on their own general preference (i.e., pretzels as stated at baseline). Finally, it is possible that the experimenter telling children that they liked pretzels, prior to the child being asked to predict the experimenter's preference, boosted children's performance (i.e., made them more likely to choose pretzels as the experimenter's future preference). However, mentioning the experimenter's baseline preference for pretzels was our attempt to equalize the self and other conditions because only children who stated a baseline preference for pretzels were included in our analyses. There are several possible reasons that children performed at-chance when predicting the experimenter's future preference. First, children's chance responding could reflect that some children answered correctly because they were able to overcome current-future state conflict to predict the experimenter's future preference, and other children might have been unduly influenced by their own current state. Alternately, children may have generally been successful in overcoming their current state but confused about the experimenter's current state that resulted in children guessing. Control questions would be useful in future work to confirm children's understanding of both the experimenter's baseline preference and their current state. More generally, replicating current findings with states other than thirst, and from perspectives other than that of the experimenter, will be important in determining if similar effects exist when making future predictions in different contexts (see Martin-Ordas, 2017, for a novel paradigm to examine current-future state conflict outside the physiological domain).

Physiological states are powerful forces that can pose difficulty for thinking beyond the present to accurately forecast one's future. The results of the current study suggest that children have difficulty predicting a future state when they are experiencing a conflicting state (e.g., thirst) and that psychological distancing improves this ability (albeit to chance levels). Further, children's current state of thirst along with age has an influence on the extent to which it interferes with future reasoning. It is critical for children to develop the ability to make accurate future predictions, even when this is at odds with one's present physiological state. Failing to do so can have immediate consequences for decision making, and more long-term consequences such as distortions of one's future happiness, financial security, and health (Gilbert et al., 2002; Gilbert, Pinel, Wilson, Blumberg, \& Wheatley, 1998; Loewenstein, 2000). Thus, is it important to recognize how one's current environment, mood, or physiological state can impact future decision making (e.g., Loewenstein, 2000). Our findings suggest that adopting the perspective of another individual may be a useful technique to mitigate the effects of a salient current state on episodic foresight, although it does not seem to result in near perfect accuracy in childhood. Future research should continue to examine this phenomenon with new paradigms and the use of self-distancing as a potential strategy that children could use to improve future thinking when current and future states are at odds.

\section{ACKNOWLEDGEMENTS}

The authors wish to thank the children and families who participated in this research as well as several research assistants who helped collect the data. Preparation of the manuscript was partially supported by an NSERC Discovery Grant to C. E. V. M.

\section{ORCID}

Tessa R. Mazachowsky (D) https://orcid.org/0000-0001-7736-3684

Caitlin E.V. Mahy (D) https://orcid.org/0000-0003-0925-611X

\section{REFERENCES}

Atance, C. M., \& Jackson, L. K. (2009). The development and coherence of future-oriented behaviors during the preschool years. Journal of Experimental Child Psychology, 102, 379-391. https://doi.org/10.1016/j.jecp.2009.01.001

Atance, C. M., \& Meltzoff, A. N. (2005). My future self: Young children's ability to anticipate and explain future states. Cognitive Development, 20, 341-361. https://doi.org/10.1016/j.cogdev.2005.05.001

Atance, C. M., \& Meltzoff, A. N. (2006). Preschoolers' current desires warp their choices for the future. Psychological Science, 17, 583-587. https://doi.org/10.1111/j.1467-9280.2006.01748.x 
Bauckham, G., Lambert, R., Atance, C., Davidson, P. S., Taler, V., \& Renoult, L. (2018). Predicting our own and others' future preferences: The role of social distance. Quarterly Journal of Experimental Physiology, 72, 634-642. https://doi.org/ 10.1177/1747021818763573

Bélanger, M. J., Atance, C. M., Varghese, A. L., Nguyen, V., \& Vendetti, C. (2014). What will I like best when I'm all grown up? Preschoolers' understanding of future preferences. Child Development, 85, 2419-2431. https://doi.org/10.1111/ cdev.12282

Bischof-Kohler, D. (1985). Zur phyogenese menschlicher motivation [On the phylogeny of human motivation]. In L. H. Eckensberger, \& E. D. Lantermann (Eds.), Emotion und reflexivitut [emotion and reflexivity] (pp. 3-47). Vienna: Urban \& Schwarzenberg.

Cheke, L. G., \& Clayton, N. S. (2019). What is the role of episodic foresight in planning for future needs? Theory and two experiments. Quarterly Journal of Experimental Psychology, XXX(XXX), XXX-XXX. https://doi.org/10.1177/ 1747021818820808

Gilbert, D. T., Gill, M. J., \& Wilson, T. D. (2002). The future is now: Temporal correction in affective forecasting. Organizational Behavior and Human Decision Processes, 88, 430-444. https://doi.org/10.1006/obhd.2001.2982

Gilbert, D. T., Pinel, E. C., Wilson, T. D., Blumberg, S. J., \& Wheatley, T. P. (1998). Immune neglect: A source of durability bias in affective forecasting. Journal of Personality and Social Psychology, 75, 617-638. https://doi.org/10.1037/00223514.75.3.617

Grossmann, I., \& Kross, E. (2014). Exploring Solomon's Paradox: Self-distancing eliminates the self-other asymmetry in wise reasoning about close relationships in younger and older adults. Psychological Science, 25, 1571-1580. https://doi.org/ $10.1177 / 0956797614535400$

Hudson, J. A., Mayhew, E. M., \& Prabhakar, J. (2011). The development of episodic foresight: Emerging concepts and methods. Advances in Child Development and Behavior, 40, 95-137. https://doi.org/10.1016/B978-0-12-3864918.00003-7

Kramer, H. J., Goldfarb, D., Tashjian, S. M., \& Lagattuta, K. H. (2017). "These pretzels are making me thirsty": Older children and adults struggle with induced-state episodic foresight. Child Development, 88, 1554-1562. https://doi.org/10.1111/ cdev. 12700

Kross, E., \& Grossmann, I. (2012). Boosting wisdom: Distance from the self enhances wise reasoning, attitudes, and behavior. Journal of Experimental Psychology: General, 141, 43-48. https://doi.org/10.1037/a0024158

Lee, W. S., \& Atance, C. M. (2016). The effect of psychological distance on children's reasoning about future preferences. PLoS ONE, 11, 1-18. https://doi.org/10.1371/journal.pone.0164382

Loewenstein, G. (1996). Out of control: Visceral influences on behavior. Organizational Behavior and Human Decision Processes, 65, 272-292. https://doi.org/10.1006/obhd.1996.0028

Loewenstein, G. (2000). Emotions in economic theory and economic behavior. The American Economic Review, 90, 426-432. https://doi.org/10.1257/aer.90.2.426

Maestro, B. (1986). The perfect picnic. Racine, WI: Western Publishing Company, Inc.

Mahy, C. E. V. (2016). Young children have difficulty predicting future preferences in the presence of a conflicting physiological state. Infant and Child Development, 25, 325-338. https://doi.org/10.1002/icd.1930

Mahy, C. E. V., Grass, J., Wagner, S., \& Kliegel, M. (2014). These pretzels are going to make me thirsty tomorrow: Differential development of hot and cool episodic foresight in early childhood. British Journal of Developmental Psychology, 32, 65-77. https://doi.org/10.1111/bjdp.12023

Martin-Ordas, G. (2017). 'Will I want these stickers tomorrow?' Preschoolers' ability to predict current and future needs. British Journal of Developmental Psychology, 35, 568-581. https://doi.org/10.1111/bjdp.12195

Moses, L. J., Coon, J. A., \& Wusinich, N. (2000). Young children's understanding of desire formation. Developmental Psychology, 36, 77-90. https://doi.org/10.1037/0012-1649.36.1.77

Nisbett, R. E., \& Kanouse, D. E. (1969). Obesity, food deprivation, and supermarket shopping behavior. Journal of Personality and Social Psychology, 12, 289-294. https://doi.org/10.1037/h0027799

Prencipe, A., \& Zelazo, P. D. (2005). Development of affective decision making for self and other: Evidence for the integration of first-and third-person perspectives. Psychological Science, 16, 501-505. https://doi.org/10.1111/j.09567976.2005.01564.x

Pronin, E., Olivola, C. Y., \& Kennedy, K. A. (2008). Doing unto future selves as you would do unto others: Psychological distance and decision making. Personality and Social Psychology Bulletin, 34, 224-236. https://doi.org/10.1177/ 0146167207310023 
Russell, J., Alexis, D., \& Clayton, N. (2010). Episodic future thinking in 3- to 5-year-old children: The ability to think of what will be needed from a different point of view. Cognition, 114, 56-71. https://doi.org/10.1016/j.cognition.2009.08.013

Suddendorf, T., \& Busby, J. (2005). Making decisions with the future in mind: Developmental and comparative identification of mental time travel. Learning and Motivation, 36, 110-125. https://doi.org/10.1016/j.Imot.2005.02.010

Suddendorf, T., Nielsen, M., \& von Gehlen, R. (2011). Children's capacity to remember a novel problem and to secure its future solution. Developmental Science, 14, 26-33. https://doi.org/10.1111/j.1467-7687.2010.00950.x

White, R. E., \& Carlson, S. M. (2016). What would Batman do? Self-distancing improves executive function in young children. Developmental Science, 19, 419-426. https://doi.org/10.1111/desc.12314

Ziegler, F. V., \& Tunney, R. J. (2012). Decisions for others become less impulsive the further away they are on the family tree. PLoS ONE, 7, e49479. https://doi.org/10.1371/journal.pone.0049479

How to cite this article: Mazachowsky TR, Koktavy C, Mahy CEV. The effect of psychological distance on young children's future predictions. Inf Child Dev. 2019;28:e2133. https://doi.org/10.1002/icd.2133 